\title{
Seasonal abundance of spider mite Tetranychus urticae Koch on vegetable and ornamental plants in Rajshahi
}

\author{
Minhajul Haque, Tamanna Islam, Najmoon Naher and Mohd. Mainul Haque \\ Department of Zoology, Rajshahi University, Rajshahi-6205
}

\begin{abstract}
The seasonal abundance of spider mite T. urticae on twenty vegetable and twenty four ornamental plants in Rajshahi was conducted during August 2010 to January 2011. The mite was present on joscpks coat, kathua, lady's finger, cucumber, brinjal, tomato, bottle gourd, bean, loofah, spinach, bitter melon, pointed gourd, radish, and cowpea but no mite was recorded on other six plants. But all the ornamental plants bagan-bilash, bottlebrush, nayantara, morog-jhuti, hasnahena, chandramallika, dalia, krisno-chura, togor, joba, madhobilata, rongon, beli, daisy, sondhamaloti, kamini, musanda, rakto-karobi, shewli, kathgolap, rajanigandha, golap, ganda, zinia contained mites. The pick population was prevailed during August. The increase of mite number is directly related with the increase of temperature.
\end{abstract}

\section{Introduction}

Mites have a worldwide distribution and causes serious damage to livestock, agricultural crops, ornamental plants and stored products. They also bring sickness and death to man; or they may be parasites, predators or saprophytes destroying animals, plants, or their products and adversely affecting man or his possessions (Johnson and Layon, 1991). Particularly two spotted spider mite has been reported to attack about 1200 species of plant (Zhang, 2003), of which more than 150 are economically important (Jeppson et al., 1997; Xie et al., 2006), such as vegetables and ornamental plants, including corn, cotton, cucumber, beans, tomato, eggplant, peppers and rose (Helle and Sabelis, 1985a,b; Navajas, 1998; Aucejo et al., 2003). It is common in green house where it is an important pest of vegetables, fruits, cut flowers and ornamental plants (Johnson and Lyon, 1991).

Several works have yet been done on mite pests all over the world. A few works have also been done in Bangladesh among which, Biswas et al., 2004; Haque et al., 2004, 2006, 2007; Haque, 2006, 2008; Naher et al., 2005, 2006,2008; Parvin and Haque, 2008; Islam et al., 2008 are important. However, there are limited number of reports of this mite on common vegetable and ornamental plants. The attempts were made to explore the presence and status of this mite on few common vegetable and ornamental plants in Rajshahi region.

\section{Materials and methods}

For the exploration of two spotted spider mites, twenty common vegetable and twenty four ornamental plants in Rajshahi City Corporation area were selected. The vegetable plants were Indian spinach (Basella alba), joscpks coat (Amaranthus gangetica), drumstick tree (Moringa concanensis), kathua (Amaranthus sp.), pointed gourd (Trichosanthes dioica), sessile joyweed (Alternanthera sessili), swamp cabbage (Ipomoea aquatica), lady's finger (Ablemoscus esculentus), cucumber (Cucumis sativus), brinjal (Solanum melongena), tomato (Lycopersicon lycopersicum), bitter melon (Monordica charantia), bottle gourd (Lagenaria vulgaris), spinach (Spinacia oleracea), spiny amaranth (Amaranthus spinosus), bean (Lablab purpureus), cowpea (Vigna sinensis), angled loofah (Luffa acutangula), radish (Raphanus sativus), coco-yam (Colocasia esculenta) and ornamental plants were baganbilash (Bougainvillea spectabilis), bottlebrush (Callistemon), nayantara (Catharanthus roseus), morog-jhuti (Celosia cristata), hasnahena (Cestrum nocturnum), chandramallika (Chrysanthemum), dalia (Dahlia), krisno-chura (Delonex regia), togor (Ervatamia divaricata), joba (Hibiscus rosa-sinensis), madhobilata (Hiptage benghalensis), rongon (Ixora coccinea), beli (Jasminum sambac), daisy (Leucanthemum vulgare), sondhamaloti (Mirabilis jalap), kamini (Murraya paniculata), musanda (Mussaenda), rakto-karobi (Nerium oleander), shewli (Nyctanthes arbortristis), kath-golap (Plumeria), rajanigandha (Polianthes tuberose), golap (Rosa $s p$.), ganda (Tagetes erecta) and zinia (Zinnia elegans).

In order to observe the abundance of two spotted spider mites on the vegetables and ornamental plants four places (Choddopaya, Binodpur Bazar, Meherchondi and Budhpara) in Rajshahi City Corporation were selected and the samples were collected from the selected plants during four/five days in a week. During each sampling leaves viz. old, mature and young were collected from five vegetable and five ornamental plants. Altogether, six leaves (2 old, 2 mature, 2 young) were taken from each plant. Sampling was done during August 2010 to January 2011. The leaves were 
collected randomly and carried to the laboratory using separate polythene bags. The total number of mites on the leaves were counted with the aid of sterieo-binocular microscope by applying counting and removing technique i.e., counted mites were removed by a camel hair brush. Daily field temperature, relative humidity and rainfall were recorded during the study period.

\section{Results and Discussion}

Nine vegetable plants viz. joscpks coat, kathua, lady's finger, cucumber, brinjal, tomato, bottle gourd, bean and loofah contained the highest number of mite per leaf during August (Table 1). The remaining of vegetable plant spinach had the highest population in October, cowpea in November, pointed gourd and radish in December and bitter melon in January respectively.

Table 1. Average number of spider mite on leaves on different vegetable and ornamental plants during August 2010 to January 2011.

\begin{tabular}{|c|c|c|c|c|c|c|}
\hline \multirow[t]{2}{*}{ Host plants } & \multicolumn{6}{|c|}{ Mite number per leaf } \\
\hline & August 2010 & September 2010 & October 2010 & November 2010 & December 2010 & January 2011 \\
\hline Indian spinach & - & - & - & - & - & - \\
\hline Joscpks coat & $15.11 \pm 0.90$ & $10.67 \pm 1.27$ & $7.92 \pm 0.52$ & $6.83 \pm 0.42$ & $4.67 \pm 0.58$ & $4.75 \pm 0.45$ \\
\hline Drumstick tree & - & - & - & - & - & - \\
\hline Kathua & $16.72 \pm 0.31$ & $10.42 \pm 0.31$ & $10.67 \pm 1.32$ & $5.67 \pm 0.67$ & $3.67 \pm 0.51$ & $3.95 \pm 0.90$ \\
\hline Pointed gourd & $18.00 \pm 1.34$ & $14.54 \pm 2.04$ & $17.38 \pm 3.50$ & $18.56 \pm 1.79$ & $19.79 \pm 2.30$ & $13.09 \pm 1.88$ \\
\hline Sessile joyweed & - & - & - & - & - & - \\
\hline Swamp cabbage & - & - & - & - & - & - \\
\hline Lady's finger & $16.44 \pm 1.29$ & $13.08 \pm 0.92$ & $10.50 \pm 0.82$ & $9.06 \pm 0.96$ & $5.93 \pm 0.44$ & $4.38 \pm 0.66$ \\
\hline Cucumber & $20.50 \pm 1.67$ & $14.72 \pm 1.79$ & $10.58 \pm 0.31$ & $8.61 \pm 0.36$ & $5.63 \pm 0.60$ & $5.11 \pm 0.49$ \\
\hline Brinjal & $22.09 \pm 0.09$ & $14.20 \pm 0.96$ & $12.63 \pm 0.61$ & $10.61 \pm 0.11$ & $7.88 \pm 0.78$ & $4.79 \pm 0.66$ \\
\hline Tomato & $13.92 \pm 0.25$ & $11.17 \pm 1.01$ & $9.33 \pm 1.08$ & $6.89 \pm 0.62$ & $7.21 \pm 0.93$ & $4.88 \pm 0.27$ \\
\hline Bitter melon & $1.09 \pm 1.09$ & $0.58 \pm 0.25$ & $4.67 \pm 0.89$ & $4.39 \pm 1.00$ & $5.33 \pm 0.10$ & $5.78 \pm 2.04$ \\
\hline Bottle gourd & $32.50 \pm 1.00$ & $22.78 \pm 2.41$ & $15.28 \pm 0.31$ & $20.67 \pm 2.06$ & $17.33 \pm 2.15$ & $10.45 \pm 0.93$ \\
\hline Spinach & $0.75 \pm 0.75$ & $0.06 \pm 0.06$ & $8.72 \pm 4.08$ & $0.39 \pm 0.39$ & $3.58 \pm 0.85$ & $1.33 \pm 0.59$ \\
\hline Spiny amaranth & - & - & - & - & - & - \\
\hline Bean & $25.42 \pm 2.42$ & $19.38 \pm 1.43$ & $13.92 \pm 0.90$ & $10.39 \pm 0.82$ & $9.17 \pm 0.85$ & $6.13 \pm 0.49$ \\
\hline Cowpea & $11.50 \pm 6.69$ & $4.00 \pm 0.62$ & $8.50 \pm 0.87$ & $17.67 \pm 1.80$ & $14.78 \pm 4.40$ & $8.22 \pm 0.59$ \\
\hline Angled loofah & $22.50 \pm 0.86$ & $13.83 \pm 0.71$ & $10.84 \pm 2.17$ & $9.00 \pm 1.40$ & $8.39 \pm 0.58$ & $7.83 \pm 0.29$ \\
\hline Radish & - & $0.00 \pm 0.00$ & $1.04 \pm 0.40$ & $5.67 \pm 1.25$ & $12.13 \pm 1.31$ & $7.71 \pm 2.69$ \\
\hline Coco-yam & - & - & - & - & - & - \\
\hline Bagan-bilash & $0.415 \pm 0.25$ & $0.39 \pm 0.056$ & $0.66 \pm 0.24$ & $0.91 \pm 0.08$ & $0.627 \pm 0.02$ & $0.66 \pm 0.24$ \\
\hline Bottlebrush & $0.58 \pm 0.08$ & $0.67 \pm 0.00$ & $0.37 \pm 0.14$ & $0.67 \pm 0.33$ & $0.33 \pm 0.17$ & $0.83 \pm 0.12$ \\
\hline Nayantara & $1.50 \pm 0.35$ & $0.83 \pm 0.49$ & $0.82 \pm 0.09$ & $1.17 \pm 0.00$ & $1.17 \pm 0.00$ & $0.50 \pm 0.28$ \\
\hline Morog-jhuti & $0.22 \pm 0.14$ & $0.67 \pm 0.00$ & $0.67 \pm 0.00$ & $0.00 \pm 0.00$ & $0.71 \pm 0.00$ & $0.67 \pm 0.06$ \\
\hline Hasnahena & $0.50 \pm 0.09$ & $0.78 \pm 0.10$ & $0.89 \pm 0.14$ & $0.67 \pm 0.29$ & $0.75 \pm 0.08$ & $0.59 \pm$ \\
\hline Chandramallika & $0.46 \pm 0.08$ & $0.78 \pm 0.54$ & $0.75 \pm 0.26$ & $0.58 \pm 0.24$ & $0.58 \pm 0.04$ & $0.78 \pm 0.05$ \\
\hline Dalia & $0.27 \pm 0.27$ & $1.17 \pm 0.00$ & $1.00 \pm 0.09$ & $1.11 \pm 0.15$ & $0.89 \pm 0.19$ & $0.25 \pm 0.14$ \\
\hline Krisno-chura & $0.14 \pm 0.42$ & $0.00 \pm 0.00$ & $0.00 \pm 0.00$ & $0.05 \pm 0.05$ & $0.00 \pm 0.00$ & $0.1 \pm 0.17$ \\
\hline Togor & $1.38 \pm 0.52$ & $0.95 \pm 0.14$ & $2.00 \pm 0.14$ & $0.46 \pm 0.17$ & $0.88 \pm 0.24$ & $0.62 \pm 0.18$ \\
\hline Joba & $0.79 \pm 0.45$ & $0.94 \pm 0.19$ & $1.16 \pm 0.09$ & $1.39 \pm 0.10$ & $1.12 \pm 0.23$ & $0.66 \pm 0.25$ \\
\hline Madhobilata & $0.50 \pm 0.49$ & $1.11 \pm 0.14$ & $0.77 \pm 0.14$ & $0.83 \pm 0.16$ & $1.16 \pm 0.14$ & $0.66 \pm 0.09$ \\
\hline Rongon & $0.75 \pm 0.07$ & $1.00 \pm 0.09$ & $1.33 \pm 0.09$ & $0.91 \pm 0.08$ & $0.62 \pm 0.20$ & $0.55 \pm 0.29$ \\
\hline Beli & $1.10 \pm 0.39$ & $1.44 \pm 0.69$ & $0.72 \pm 0.14$ & $0.33 \pm 0.16$ & $1.15 \pm 0.18$ & $0.50 \pm 0.00$ \\
\hline Daisy & $1.00 \pm 0.32$ & $0.25 \pm 0.20$ & $0.54 \pm 0.04$ & $0.33 \pm 0.00$ & $0.66 \pm 0.16$ & $0.45 \pm 0.26$ \\
\hline Sondhamaloti & $0.77 \pm 0.05$ & $0.83 \pm 0.33$ & $0.83 \pm 0.00$ & $0.83 \pm 0.00$ & $0.66 \pm 0.16$ & $0.83 \pm 0.00$ \\
\hline Kamini & $0.83 \pm 0.09$ & $0.50 \pm 0.00$ & $0.83 \pm 0.09$ & $0.71 \pm 0.14$ & $0.94 \pm 0.05$ & $0.44 \pm 0.14$ \\
\hline Musanda & $2.11 \pm 0.24$ & $1.22 \pm 0.10$ & $1.63 \pm 0.15$ & $1.58 \pm 0.08$ & $1.17 \pm 0.28$ & $0.58 \pm 0.41$ \\
\hline Rakto-karobi & $1.17 \pm 0.44$ & $1.00 \pm 0.16$ & $0.72 \pm 0.14$ & $0.99 \pm 0.16$ & $1.16 \pm 0.09$ & $0.50 \pm 0.00$ \\
\hline$\overline{\text { Shewli }}$ & $0.71 \pm 0.35$ & $0.94 \pm 0.10$ & $0.95 \pm 0.04$ & $0.00 \pm 0.00$ & $0.83 \pm 0.09$ & $0.47 \pm 0.33$ \\
\hline Kath-golap & $1.00 \pm 0.16$ & $1.61 \pm 0.14$ & $1.67 \pm 0.00$ & $1.33 \pm 0.16$ & $1.27 \pm 0 . ` 0$ & $1.25 \pm 0.57$ \\
\hline Rajanigandha & $1.33 \pm 0.66$ & $0.70 \pm 0.20$ & $0.24 \pm 0.16$ & $0.66 \pm 0.16$ & $0.66 \pm 0.06$ & $0.25 \pm 0.24$ \\
\hline Golap & $0.83 \pm 0.23$ & $1.33 \pm 0.69$ & $0.44 \pm 0.21$ & $0.61 \pm 0.05$ & $0.58 \pm 0.05$ & $0.61 \pm 0.05$ \\
\hline Ganda & $0.58 \pm 0.08$ & $0.45 \pm 0.14$ & $0.45 \pm 0.17$ & $0.39 \pm 0.11$ & $0.16 \pm 0.00$ & $0.41 \pm 0.13$ \\
\hline Zinia & $0.08 \pm 0.07$ & $0.55 \pm 0.05$ & $0.61 \pm 0.05$ & $0.69 \pm 0.16$ & $0.83 \pm 0.06$ & $0.83 \pm 0.33$ \\
\hline
\end{tabular}

In case of ornamental plants nayantara, krisnochura, daisy, musanda, rakto-karobi, rajanigandha, ganda, had the highest number of mite during August; chandramallika, dalia, beli, sondhamaloti, golap in September; hasnahena, togor, rongon, shewli, kath-golap in October; 
bagan-bilash and joba in November; morogjhuti, madhobilata, kamini, zinia, in December and bottlebrush in January respectively. The ornamental plants contained the lower number of mite in comparison to vegetable plants.

The impacts of environmental factors on mite population on different types of vegetables and ornamental plants were studied and the calculated ' $r$ ' values are presented in Table 2 . In vegetable plants temperature had direct positive impact on mite population on joscpks coat, kathua, lady's finger, cucumber, brinjal, tomato, bottle gourd, bean, angled loofah, rongon, daisy, musand and negative impact on bitter melon, radish, morog-jhuti and zinnia. In case of brinjal and sondhamaloti mite number increased significantly with the increase of relative humidity but on radish mite number decreased significantly with the increase of relative humidity.

Table 2: The values of coefficient of co-relation $(r)$ between mite number and different environment factors.

\begin{tabular}{|c|c|c|c|c|c|c|c|}
\hline \multicolumn{4}{|c|}{ Vegetable plants } & \multicolumn{4}{|c|}{ Ornamental plants } \\
\hline \multirow{2}{*}{ Host plant } & \multicolumn{3}{|c|}{$r$ values with } & \multirow{2}{*}{ Host plant } & \multicolumn{3}{|c|}{$r$ values with } \\
\hline & Temp $\left({ }^{\circ} \mathrm{C}\right)$ & Rh (\%) & $\mathrm{RF}(\mathrm{mm})$ & & $\operatorname{Temp}\left({ }^{\circ} \mathrm{C}\right)$ & $\mathrm{Rh}(\%)$ & $\mathrm{RF}(\mathrm{mm})$ \\
\hline Indian spinach & - & - & - & Bagan-bilash & -0.12 & -0.15 & 0.30 \\
\hline Joscpks coat & $0.82^{\star \star \star}$ & 0.27 & -0.02 & Bottlebrush & -0.17 & -0.08 & 0.19 \\
\hline Drumstick tree & - & - & - & Nayantara & 0.36 & -0.06 & -0.02 \\
\hline Kathua & $0.78^{\star \star \star}$ & 0.27 & 0.27 & Morog-jhuti & $-0.54^{*}$ & 0.15 & -0.38 \\
\hline Pointed gourd & 0.13 & -0.27 & 0.13 & Hasnahena & -0.07 & -0.09 & 0.08 \\
\hline Sessile joyweed & - & - & - & Chandramallika & -0.21 & -0.39 & -0.10 \\
\hline Swamp cabbage & - & - & - & Dalia & 0.28 & 0.26 & -0.30 \\
\hline Lady's finger & $0.90^{\star \star \star}$ & 0.36 & 0.22 & Krisno-chura & 0.06 & -0.07 & -0.20 \\
\hline Cucumber & $0.83^{\star \star *}$ & -0.04 & $0.53^{*}$ & Togor & 0.12 & 0.14 & -0.09 \\
\hline Brinjal & $0.85^{\star \star \star}$ & $0.53^{* *}$ & 0.23 & Joba & -0.00 & 0.29 & -0.21 \\
\hline Tomato & $0.78^{\star \star \star}$ & 0.28 & 0.10 & Madhobilata & -0.06 & 0.12 & -0.05 \\
\hline Bitter melon & $-0.60^{*}$ & -0.28 & -0.39 & Rongon & $0.57^{*}$ & 0.25 & 0.38 \\
\hline Bottle gourd & $0.61^{* *}$ & 0.46 & $0.49^{*}$ & Beli & 0.15 & -0.27 & -0.05 \\
\hline Spinach & 0.13 & 0.18 & 0.24 & Daisy & $0.42^{*}$ & 0.13 & 0.41 \\
\hline Spiny amaranth & - & - & - & Sondhamaloti & 0.11 & $0.61^{*}$ & -0.20 \\
\hline Bean & $0.86^{\star \star \star}$ & 0.29 & $0.53^{\star \star}$ & Kamini & 0.06 & -0.15 & 0.00 \\
\hline Cowpea & -0.17 & 0.11 & -0.29 & Musanda & $0.59 *$ & 0.18 & -0.04 \\
\hline Angled loofah & $0.71^{* * *}$ & 0.22 & 0.15 & Rakto-karobi & 0.38 & 0.20 & -0.10 \\
\hline Radish & $-0.56^{\star}$ & $-0.58^{*}$ & -0.14 & Shewli & 0.28 & 0.28 & -0.24 \\
\hline \multirow[t]{5}{*}{ Coco-yam } & - & - & - & Kath-golap & 0.19 & 0.15 & 0.20 \\
\hline & & & & Rajanigandha & 0.38 & -0.21 & 0.16 \\
\hline & & & & Golap & 0.37 & -0.30 & -0.27 \\
\hline & & & & Ganda & 0.15 & -0.13 & -0.28 \\
\hline & & & & Zinia & $-0.47^{*}$ & 0.06 & -0.10 \\
\hline
\end{tabular}

* $=\mathrm{P}<0.05,{ }^{* *}=\mathrm{P}<0.01,{ }^{* * *}=\mathrm{P}<0.001$

In case of ornamental plants mite population significantly increased with the increase of temperature on rongon, daisy and musanda but decrease with the increase of temperature on morog-jhuti and zinia. The relative humidity was found to affect the mite populations only on sondhamaloti. Temperature has been the most extensively studied of all weather factors that bear on mite population and it appears to have the most over all influence. Low temperature can cause reductions in winter populations. High mortality also occurs when unseasonably low temperature follows worm weather in early spring.

Gotoh (1997) studied the population of T. urticae in four different Japanese pear orchards in Ibaraki prefecture and found the seasonal prevalence's of mite populations then similar, but their abundances varied greatly. He observed two types of seasonal population trends in $T$. urticae. In one pear orchard there was a population peak from September to early October and in the other there were two peaks in July and September.

Nahar (2005) made extensive survey and recorded higher number of $T$. urticae in April and August. The preferred temperature for the rapid development of $T$. urticae is 25 to $27^{\circ} \mathrm{C}$ (Jeppson et al., 1975). The impact of temperature on the mite population shows the positive significant effect on most of the vegetable plants. In case of the host $M$. charanta (bitter melon) and $R$. saativus (radish) the temperature exerted the negative impact on mite number. As these are winter crops and plants did not develop sufficiently due to hot temperature not fit to supply the food for the pest animal, the population reduced due to rise of temperature. 


\section{References}

Aucejo, S., Foo, M., Gimeno, E., Gomez, A., Montort, R., Predes, E., Ramis, M., Ripolles, J. L. \& Tirado, V. 2003. Management of Tetranychus urticae in Citrus in Spain acarofauna associated to weed. IOBC/WPRS Bull. 26: 213-220.

Biswas, G.C., Islam, W. \& Haque, M.M. 2004. Seasonal abundance of spider mites (Acari: Tetranychidae) on some economic plants in Rajshahi. J. bio. Sci. Rajshahi University, Bangladesh. 7(12): 1-6.

Gotoh, T. 1997. Annual life cycles of population of the two spotted spider mite, Tetranychus urticae Koch (Acari:Tetranychidae) in four Japanese pear orchards. App. Ent. Zool. 2001. 32(1): 207-316.

Haque, M. M., Naher, N. \& Kawai, A. 2004. Tomato russet mite population \& searching of its predator on open field tomato plant. Bangladesh j. entomol; 14 (2): 7-17.

Haque, M.M. 2006. Population growth and development of two-spotted spider mite, Tetranychus urticae Koch (Acari: Tetranychidae) on four host plants. Rajshahi University Studies. Part B. 34: 205-210.

Haque, M.M., Islam, M.M., Das. B.C. \& Naher, N. 2006. Seasonal abundance of Tetranychus urticae Koch (Acari: Tetranychidae) infesting rose plants and effects of some pesticides on it. J. Asiat. Soc. Bangladesh Sci. 32(1): (75-80).

Haque, M.M., Wahab, A., Naher, N. \& Begum, A. 2007. Developmental stages of red spider mite, Oligonychus coffeae Neither Koch (Acari: Tetranychidae) infesting rose.. Univ. j. zool. 26: (71-72).

Helle, W.\& Sabelis, M.W. [eds.].1985a. Spider mites: Their biology, natural enemies and control. Volume 1 Part A. Elsevier, Amsterdam. 406pp.

Helle, W. \& Sabelis, M.W. [eds.].1985b. Spider mites: Their biology, natural enemies and control. Volume 1 Part B. Elsevier, Amsterdam. 458 pp.

Islam, M.T., Haque, M.M., Naher, N. \& Parween, S. 2008. Effect of plant materials on the developmental stages of two spotted Spider mite Tetranychus urticae Koch (Acari: Tetranychidae). J. bio. Sci. 16: 121-124.
Jeppson, L.R., Keifer, H.H. \& Baker, E.W. 1975. Mites Injurious to Economic Plants. University of California Press, Berkeley, CA. 458 pp.

Jeppson, L.R., Keifer, H.H. \& Baker, E.W. 1997. Mites Injurious to Economics Plants. University of California Press, Berkeley, CA. 458 pp.

Johnson, W.T. \& Layon, H.H. 1991. Insects that feed on trees and shrubs. Comstock Publishing and Cornell University press, Ithaca, NY. 416 pp.

Nahar, N. 2005. Integrated management of two sportted spider mite infesting beans. Ph.D thesis. Rajshahi University, Banglaesh. 157 pp.

Naher, N., Islam, M. W., Khalequzzaman, M. \& Haque, M. M. 2008 Study on the developmental stages of spider mite (Tetranychus urticae Koch) infesting country bean. J. bio.sci. 16: 109-114.

Naher, N., Islam, T., Haque, M.M. \& Parween, S. 2006. Effects of native plants and IGRs on the development of Teiranychus urticae Koch (Acari: Tetranychidae). Univ. j. zool. 25: 19-22.

Naher, N., Islam, W. \& Haque, M. M. 2005. Predation of three predators on two-spotted spider mite, Tetranychus urticae Koch (Acari: Tetranychidae). Journal of Life \& Earth Science. 1: 1-4.

Navajas, M. 1998. Host plant association in the spider mite Tetranychus urticae (Acari Tetranychidae): in sights from molecular phylogeography. Exp. Appl.Acarol .22: 201-214.

Parvin, M.M. \& Haque, M.M. 2008. Control of twospotted spider mite, Tetranychus articae Koch (Acari: Tetranychidae) by predators on potted plants. Univ. j. zool. 27: 51-54.

Xie, L., Miao, H. \& Xiao-Yue Hong X-Y. 2006. The two spotted spider mite Tetranychus urticae Koch and the carmine spider mite Tetranychus cinnabarinus (Boisduval) in China mixed in their Wolbachia phylogenetic tree. Zoolaxa 1166: 33-46.

Zhang, Z. 2003. Mites of Greenhouses. CABI Publishing Oxon, UK. 244 pp. 